\title{
Detection of Blood Lipids, Homocysteine and Endothelin in Patients with Hypothyroidism and Subclinical Hypothyroidism and Its Clinical Significance
}

\author{
Li Hongyan ${ }^{1,2, *}$, Xu Qian ${ }^{1,2}$, Liu Jing ${ }^{3}$, Li Juan ${ }^{3}$ \\ ${ }^{1}$ Department of Medical Technology of Cangzhou Medical College, Cangzhou, China \\ ${ }^{2}$ Cangzhou Thyroid Disease Engineering Research Center, Cangzhou, China \\ ${ }^{3}$ Laboratory Department of Cangzhou Traditional Chinese Medicine-Western Hospital, Cangzhou, China
}

Email address:

czyzjyk@126.com (Li Hongyan)

${ }^{*}$ Corresponding author

\section{To cite this article:}

Li Hongyan, Xu Qian, Liu Jing, Li Juan. Detection of Blood Lipids, Homocysteine and Endothelin in Patients with Hypothyroidism and Subclinical Hypothyroidism and Its Clinical Significance. American Journal of Clinical and Experimental Medicine.

Vol. 7, No. 1, 2019, pp. 31-34. doi: 10.11648/j.ajcem.20190701.14

Received: February 18, 2019; Accepted: April 17, 2019; Published: April 29, 2019

\begin{abstract}
Objective To discuss the changes of blood lipids, homocysteine (Hcy) and endothelin (ET) levels in patients with hypothyroidism and subclinical hypothyroidism, and to explore their clinical significance. Methods The authors choose 255 cases of hypothyroidism, 274 cases of subclinical hypothyroidism and 130 cases of the control group to test serum TC, TG, HDL, LDL, LPa, Hcy and ET. The correlation were analyzed. Results The level of TC, TG, LDL of hyperthyroidism group and subclinical hyperthyroidism group are higher than the control group, HDL is lower than the control group. The level of TC, TG, LDL of hyperthyroidism group is higher than subclinical hyperthyroidism group, HDL is lower than subclinical hyperthyroidism group. The level of Hcy of hyperthyroidism group and subclinical hyperthyroidism group are higher than the control group. The level of Hcy of hyperthyroidism group is higher than subclinical hyperthyroidism group. The level of ET of hyperthyroidism group is lower than the control group and subclinical hyperthyroidism group. Conclusions All of blood lipids, Hcy and ET participate in the development process of hypothyroidism and subclinical hypothyroidism which is of great value in predicting the transition of thyroid dysfunction and the occurrence of adverse consequences.
\end{abstract}

Keywords: Hypothyroidism, Subclinical Hypothyroidism, Blood Lipids, Hcy, ET

\section{Introduction}

As an endemic disease in Cangzhou and its surrounding areas, thyroid diseases have attracted much attention for many years. Hypothyroidism is a common endocrine disease in clinic, and the incidence of hypothyroidism is increasing year by year. Thyroid dysfunction can cause lipid metabolism abnormality, induce endocrine diseases such as lipid metabolism disorder [1, 2], and then lead to atherosclerosis, myocardial infarction and other serious adverse consequences. Hypothyroidism has mild clinical symptoms or no clinical symptoms. It is easy to be ignored during the clinical examination, but it may eventually develop into clinical hypothyroidism [3]. At present, the influence of hypothyroidism on blood lipid is still controversial, and the relationship between hypothyroidism and Hcy, ET are receiving more and more attention from the scholars $[4,5]$. The aim of this study was to determine the levels of serum lipids, Hcy and ET in patients with hypothyroidism and subclinical hypothyroidism, and to explore the relationship between them and the occurrence and development of hypothyroidism and subclinical hypothyroidism, so as to provide evidence for the prevention and treatment of these endemic diseases.

\section{Datas and Methods}

\subsection{General Datas}

From March 2016 to October 2018, 531 patients (203 males and 328 females) in the Department of Endocrinology of our 
hospital were selected as the experimental group. The patients were divided into two groups: 255 patients in hypothyroidism group, among them 97 males and 158 females, age 21 70 years old, averaging $42.2 \pm 11.4$ years old; And the other 276 patients in subclinical hypothyroidism group, among them 106 males and 170 females, age 21 70 years old, averaging $44.2 \pm 11.4$ years old. All cases were newly diagnosed cases, without any treatment, and diagnosed by clinic and laboratory to exclude the possible endocrine system and non-thyroid systemic diseases.

At the same time, 130 healthy people, among them 58 males and 72 females, averaging $45.3 \pm 12.5$ years old were selected as the control group. The heart, liver, kidney, lung and other important organs were normal, and the thyroid function and blood routine examination were normal. Endocrine system and non-thyroid systemic diseases were excluded.

\subsection{Methods}

Patients and healthy ones both receiving a fasting hemospasia to collect $4 \mathrm{ml}$ venous blood, then the serum was isolated by high-speed centrifugation after resting for $1 \mathrm{~h}$ for $2 \mathrm{~h}$ at room temperature. The serum thyroid function was measured by Cobas e602 automatic Electrochemiluminescence Immunoassay and its related reagents, determine the five indexes of serum thyroid function: free triiodothyronine (FT3), free thyroxine (FT4), total triiodothyronine (TT3), total thyroxine (TT4), thyroid stimulating hormone (TSH); The serum TC, TG, HDL, LDL and LPa were determined by HITACHI- 020 automatic biochemistry analyzer. Using Abbott AXSYM automatic Immunology Analyzer to determine Hcy; ET radioimmunoassay Kit purchased from Finland Orion Diagnostica Company.

\subsection{Diagnostic Standard}

1) Hypothyroidism: Possess a symptom or sign of hypothyroidism consistent with FT3 $<2.8 \mathrm{pmol} / \mathrm{L}$ and / or FT4 $<12 \mathrm{pmol} / \mathrm{L}, \quad \mathrm{TSH}>4.2 \mathrm{mIU} / \mathrm{L} ; \quad 2) \quad$ subclinical hypothyroidism: TSH $>4.2 \mathrm{~m} \mathrm{IU} \mathrm{/} \mathrm{L,} \mathrm{FT3} \mathrm{and} \mathrm{FT4} \mathrm{were} \mathrm{in}$ normal range. SPSS19.0 statistical software was used to analyze the data, the data were expressed as $\bar{x} \pm S$, he quantitative data were compared by t-test, and the correlation among the factors was analyzed by linear correlation analysis. $\mathrm{P}<0.05$ suggests that there were significant differences.

\subsection{Statistical Analysis}

SPSS19.0 statistical software was used to process the data, the metrological data were expressed as $\bar{x} \pm \mathrm{s}$, the quantitative data were compared by t-test, and the correlation among the factors was analyzed by linear correlation analysis. $\mathrm{P}<0.05$ suggests that there were significant differences.

\section{Results}

\subsection{Comparison of Blood Lipid Levels in Each Group}

TC, TG, LDL level in hypothyroidism group was higher than the control group, HDL levels were lower than the control group, and the difference was statistically significant $(\mathrm{P}<0.01)$. The TC, TG LDL levels in hypothyroidism group was higher than those in Subclinical hypothyroidism group, and the HDL level was lower than that in Subclinical hypothyroidism group. The difference was statistically significant $(\mathrm{P}<0.01)$. There was no significant difference in comparing of the level of $\mathrm{LPa}$ in each group $(\mathrm{P}>0.05)$. The results are shown in Table 1.

Table 1. Comparison of blood lipid levels in each group $(x \pm s)$.

\begin{tabular}{llll}
\hline results & $\begin{array}{l}\text { control group } \\
(\mathbf{n = 1 3 0})\end{array}$ & $\begin{array}{l}\text { Subclinical } \\
\text { hypothyroidism } \\
\text { group }(\mathbf{n = 2 7 6})\end{array}$ & $\begin{array}{l}\text { hypothyroidism } \\
\text { group }(\mathbf{n = 2 5 5})\end{array}$ \\
\hline $\mathrm{TC}(\mathrm{mmol} / \mathrm{L})$ & $4.51 \pm 0.92$ & $5.18 \pm 1.06$ & $5.53 \pm 1.10$ \\
$\mathrm{TG}(\mathrm{mmol} / \mathrm{L})$ & $1.44 \pm 0.47$ & $1.69 \pm 0.50$ & $1.90 \pm 0.53$ \\
$\mathrm{HDL}(\mathrm{mmol} / \mathrm{L})$ & $1.41 \pm 0.27$ & $1.34 \pm 0.21$ & $1.25 \pm 0.19$ \\
$\mathrm{LDL}(\mathrm{mmol} / \mathrm{L})$ & $2.97 \pm 1.01$ & $3.38 \pm 0.98$ & $3.69 \pm 1.01$ \\
$\mathrm{LPa}(\mathrm{mg} / \mathrm{L})$ & $220.37 \pm 21.46$ & $212.29 \pm 18.73$ & $230.18 \pm 22.71$ \\
\hline
\end{tabular}

\subsection{Comparison of Hcy and ET Levels in Each Group}

The levels of Hcy in hypothyroidism group and Subclinical hypothyroidism group were higher than those in control group, and the level of Hcy in hypothyroidism group was higher than that in Subclinical hypothyroidism group, the difference was statistically significant $(\mathrm{P}<0.01)$. The level of ET in hypothyroidism group was lower than that in control group and Subclinical hypothyroidism group, the difference was statistically significant $(\mathrm{P}<0.05)$. There was no significant difference in ET level between hypothyroidism group and control group $(\mathrm{P}>0.05)$. The results are shown in Table 2 .

Table 2. Comparison of Hcy and ET levels in each group ( $x \pm s)$.

\begin{tabular}{llll}
\hline results & $\begin{array}{l}\text { control } \\
\text { group } \\
(\mathbf{n}=\mathbf{1 3 0})\end{array}$ & $\begin{array}{l}\text { Subclinical } \\
\text { hypothyroidism } \\
\text { group }(\mathbf{n}=\mathbf{2 7 6})\end{array}$ & $\begin{array}{l}\text { hypothyroidism } \\
\text { group }(\mathbf{n}=\mathbf{2 5 5})\end{array}$ \\
\hline Hcy $(\mathrm{umol} / \mathrm{L})$ & $13.85 \pm 1.92$ & $18.20 \pm 2.06$ & $22.63 \pm 2.10$ \\
ET $(\mathrm{ng} / \mathrm{L})$ & $110.19 \pm 12.62$ & $104.66 \pm 12.11$ & $90.12 \pm 11.94$ \\
\hline
\end{tabular}

\subsection{The Correlation Between Thyroid Function Index and Blood Lipid, Hcy, ET in Subclinical Hypothyroidism Group}

Hcy level was negatively correlated with FT3, FT4 and ET in hypothyroidism group $(\mathrm{r}=-0.526,-0.478,-0.463, \mathrm{P}<0.01$, respectively), and it was positively correlated with TG and TC ( $\mathrm{r}=0.411, \mathrm{r}=0.371, \mathrm{P}<0.01$, respectively). There was no correlation between other indexes of blood lipid. In Subclinical hypothyroidism group, Hcy level was negatively correlated with FT3 and FT4 $(\mathrm{r}=-0.382,-0.256, \mathrm{P}<0.01$, respectively), but had no correlations with other indexes.

\section{Discussions}

The whole body of a Hypothyroidism patient is in the state of a low metabolic level, with diastolic hypertension, about $80 \%$ of hypothyroidism patients with dyslipidemia [6], making hypothyroidism patients with high incidence of cardiovascular diseases [7]. Hypothyroidism is a type of thyroid dysfunction, of all the thyroid diseases; it has a higher incidence rate [8], and are closely related to the changes in blood lipid levels [9]. 
Wu Pengfei et al [10] found that the levels of TC, TG and LDL were higher and the levels of HDL were lower in patients with hypothyroidism. Chen Xiaofang et al [4] reported that with the increase of TSH, the levels of TC, TG and LDL would increase, but the level of HDL would decrease in hypothyroidism patients. The study of Zhou Hui et al [11] shows that the levels of TC, LDL-C and APOB in subclinical hypothyroidism group were significantly higher than those in control group. My research shows that the levels of TC, TG and LDL in hypothyroidism group and subclinical hypothyroidism group were higher than those in control group, and the level of HDL was lower than that in control group, and the difference was statistically significant $(\mathrm{P}<0.01)$, which was consistent with the report listed above. It was also found that the levels of TC, TG and LDL in hypothyroidism group were higher than those in subclinical hypothyroidism group, and in the meanwhile the level of HDL lower than that in hypothyroidism group. This suggests that subclinical hypothyroidism may not having clinical symptoms, but is accompanied by changes of blood lipid, which is the pathological basis of many cardiovascular and cerebrovascular diseases [12], and may induce myocardial ischemia, leading to serious consequences, such as angina pectoris, myocardial infarction and so on. Therefore, a regular monitoring of blood lipid changes is of great value in predicting whether hypothyroidism occurs and whether it is associated with atherosclerotic heart disease.

Hcy is closely associated with some common clinical diseases [13]. It has been revealed that hyperlipidemia is an important factor leading to cardiovascular disease and stroke $[14,15,16]$, and a slight or moderate increase in the level of Hcy can lead to the occurrence of systemic atherosclerosis [17]. Other studies suggest that high Hcy is a thrombogenic agent that can cause cerebral infarction [18]. Dai Lili et al [19] reported that subclinical hypothyroidism patients had elevated Hcy. My research found that Hcy levels of both the hypothyroidism group and subclinical hypothyroidism group were higher than the control group. The level of Hcy in hypothyroidism group was negatively correlated with FT3, FT4 and ET, and positively correlated with TG and TC. The level of Hcy in subclinical hypothyroidism group was negatively correlated with FT3 and FT4. Hypothyroidism and subclinical hypothyroidism patients have lower metabolic rate less gastric acid secretion, and, decreased capacity of intestinal absorption, leading to absorption disorders of folic acid and vitamin B12. As folic acid and vitamin B12 are important coenzymes in the metabolism of Hcy, and play an important role in its transformation process, once reduced, will cause the increase of Hcy and further more, promote the growth of oxygen free radicals, damaging vascular endothelial cells, Causing atherosclerosis [20]. In addition, Hcy can elevate serum LDL, induce its oxidation, form foam cells, and then aggravate atherosclerosis.

Produced by vascular endothelial cells, ET is a 21 amino acid peptide with strong vasoconstrictive activity and low plasma concentration in physiological condition. However, when vascular endothelial cells are damaged, the release of ET increases, resulting in a series of pathophysiological changes. Zhu Yalin et al [21] found that plasma ET in untreated hypothyroidism group was significantly lower than that in control group and treatment group. Chen Xiaofang and other people ${ }^{[4]}$ reported that there was no significant correlation between ET and hypothyroidism. My research shows that the level of ET in hypothyroidism group was lower than that in control group and subclinical hypothyroidism group. There was no difference in ET level between subclinical hypothyroidism group and control group, which was consistent with the above report. It may be related to hypothyroidism patients with low hemodynamics, patients in a state of low metabolism, and reduced sympathetic nerve activity. Although subclinical hypothyroidism patients have vascular endothelial dysfunction, there is no significant difference in the level of ET because there are no significant differences between subclinical hypothyroidism and the normal effects of hypoxia-ischemia.

\section{Conclusion}

To sum up, the abnormal changes of thyroid function in hypothyroidism and subclinical hypothyroidism patients are related to hyperlipidemia, high Hcy and low ET. Regular review of these indicators for early detection, early intervention, and early treatment of hypothyroidism patients is very necessary to prevent subclinical hypothyroidism from developing into clinical hypothyroidism and atherosclerosis and other adverse illness.

\section{Fund Project}

Self-financed Project of Hebei Science and Technology Research and Development Program (No. 162777205).

Cangzhou Science and Technology Research and Development Guidance Program Project (Ref. 151302011).

\section{References}

[1] Vanessa S. Virgini, Liselotte W. Wijsman, Nicolas Rodondi, et al. Subclinical Thyroid Dysfunction and Functional Capacity Among Elderly [J]. Thyroid, 2014, 24 (2): 208-214.

[2] Chen-Hua, Xin-Ning. Clinical efficacy and changes in serum lipid index of levothyroxine in subclinical and clinical hypothyroidism [J]. Chinese Journal of Clinical Research, 2014, 6 (9):84

[3] Wiles KS, Jarvis S, Nelson-Piercy C. Are we overtreating subclinical hypothyroidism in pregnancy? [J]. BMJ, 2015, 351 h4726.

[4] Chen Xiao-fang, Xu-Bo, Feng-Kai, et al. Study on endothelin, fibrinogen and lipid levels in subclinical hypothyroidism [J]. International Journal of Laboratory Medicine, 2012, 33 (23):2828-2829.

[5] Shi Qing-jun, Dai li-li. Changes of serum resistin and homocysteine levels in patients with subclinical hypothyroidism [J]. Chinese Journal of Gerontology, 2017, 37 (15):3765-3766. 
[6] Guo Juan-juan. Effects of levothyroxine sodium combined with thyroid tablets on thyroid hormone changes in elderly patients with hypothyroidism [J]. Frontier of Medicine, 2017, 07 (13):132-133.

[7] Pan Yan-hui, Sun Yun-peng, Zhang-Wei. Effects of levothyroxine sodium combined with thyroid tablets on thyroid function and blood lipids in patients with hypothyroidism [J]. Women's Health Research, 2018 (14):39-40.

[8] Ren Yun-xia, Zhang Jun-qi, Fan Chun-yu. Clinical characteristics and prognosis of patients with coronary heart disease complicated with subclinical hypothyroidism [J]. Chinese Journal of Integrative Medicine on Cardio-Cerebrovascular Disease, 2018, 16 (10):1381-1384.

[9] Gong Ning-ning, Gong Ting-ting. Research progress on blood lipid level in subclinical hypothyroidism [J]. Scientific \& Technical Information of Gansu, 2018, 47 (05):81-85.

[10] Wu Peng-fei, Dong-Zhen. The relationship between hypothyroidism and serum levels of total homocysteine, lipid and endothelin [J]. Journal of Bethune Military Medical College, 2013, 11 (06):508-509.

[11] Zhou-Hui, Li Peng-qiu, Liu Li-mei, et al. Study on TSH and dyslipidemia in subclinical hypothyroidism [J]. Journal of China Prescription Drug, 2018, 16 (02):7-8.

[12] Qi Ying-ying. Clinical Significance of Combined Detection of Lipoprotein Alpha, Homocysteine and Routine Blood Lipids in Atherosclerosis [J]. China Medical Engineering, 2018, 26 (4): $51-53$.

[13] Hu Yao-shi, Zeng Zhi-yu. Research Progress on the Relationship between Homocysteine and Common Clinical Diseases [J]. Chinese Journal of New Clinical Medicine, 2017, $10(08): 811-815$.
[14] Guan Shi-kui, Liu-Zheng, Zhang Fa-sheng, et al. Mete analysis of the relationship between serum cystatin $\mathrm{C}$ level and coronary heart disease $[\mathrm{J}]$. Labeled Immunoassays and Clinical Medicine, 2015, 22 (10): 994-997.

[15] Chi Xin-dong, Ma-Li, He-Jia, et al. Diagnostic value of combined detection of homocysteine (Hcy), serum cystatin $\mathrm{C}$ (CysC) and urinary microalbumin (U-Malb) for early renal injury in hypertension $[\mathrm{J}]$. Chinese Journal of Laboratory diagnosis, 2017, 21 (6): 939-941.

[16] Hou Zhen-jiang, Mou Zhao-xin, Zhang Jing-yu, et al. The clinical significance in teseing serum cystatin $\mathrm{C}$ and homocysteine of subclinical thyroid dysfunction [J]. Medicine \& Philosophy (B), 2017, 38 (08):40-44.

[17] Yu-Yang, Chen Hui-yong, Sun-Chao. Effects of Danhong injection on C-reactive protein and homocysteine of patients with early lower extremity arteriosclerosis obliteran [J/OL]. World Chinese Medicine, 2018 (04):909-914

[18] Chen-Na. The effect of folic acid and Mecobenamine on 41 patients with acute cerebral infarction with high homocysteine [J]. Contemporary medicine, 2018, 24 (13): 72-74.

[19] Dai Li-li, Liu Jia-jia, Mi Yu-jing et al, Study on the correlation between subclinical thyroid dysfunction and serum resistin and homocysteine [J]. Chinese Journal of Health laboratory Technology, 2015, 25 (14):2346-2348.

[20] Wei-Yan, Jie Jia-mei, Shi Li-xin. The metabolic characteristics of lipid profile and homocysteine in the patients with subclinical hypothyroidism [J]. International Journal of Laboratory Medicine, 2013, 34 (24):3330-3331+3334.

[21] Zhu ya-lin, Huo-ying, Pan yun-long. Clinical significance of plasma atrial natriuretic factor and endothelin detection in hyperthyroidism and hypothyroidism [J]. Labeled Immunoassays and Clinical Medicine, 2005 (01):13-14+3. 